BNL-NT-07/46

RBRC-702

\title{
On the Relation Between Mechanisms for Single-Transverse-Spin Asymmetries
}

\author{
Yuji Koike \\ Department of Physics, Niigata University, Ikarashi, Niigata 950-2181, Japan \\ Werner Vogelsang \\ Physics Department, Brookhaven National Laboratory, Upton, NY 11973
}

Feng Yuan

Nuclear Science Division, Lawrence Berkeley National Laboratory, Berkeley, CA 94720 and RIKEN BNL Research Center, Building 510A, Brookhaven National Laboratory, Upton, NY 11973

\begin{abstract}
Recent studies have shown that two widely-used mechanisms for single-transverse-spin asymmetries based on either twist-three contributions or on transverse-momentum-dependent (Sivers) parton distributions become identical in a kinematical regime of overlap. This was demonstrated for the so-called soft-gluon-pole and hard-pole contributions to the asymmetry associated with a particular quark-gluon correlation function in the nucleon. In this paper, using semi-inclusive deep inelastic scattering as an example, we extend the study to the contributions by soft-fermion poles and by another independent twist-three correlation function. We find that these additional terms organize themselves in such a way as to maintain the mutual consistency of the two mechanisms for single-spin asymmetries.
\end{abstract}




\section{INTRODUCTION}

Much progress has been made in recent years in the theoretical understanding of singletransverse-spin asymmetries (SSAs) in high-energy hadronic scattering. Traditionally, two mechanisms for generating SSAs in Quantum Chromodynamics (QCD) had been identified and used in the literature. One is based on "asymmetric" transverse-momentum-dependent (TMD) parton distributions or fragmentation functions, in particular the so-called Sivers [1] and Collins [2] functions, respectively. Here, a TMD factorization is employed. The other mechanism makes use of twist-3 quark-gluon correlation functions, either in the nucleon [3-7] or in fragmentation [8], and of collinear factorization.

The recent progress came initially with the study of the factorization and universality properties of TMDs and the related spin-dependent cross sections in Refs. [9-14]. A highlight of these investigations has been the finding that the Sivers functions are not universal in the usual sense, but enter with opposite sign in semi-inclusive deep-inelastic scattering (SIDIS) and the Drell-Yan process. These studies were subsequently extended to more complicated QCD hard-scattering [15-17], which revealed a very complex general pattern of non-universality of the Sivers functions.

In addition to this, recent work [18-20] (hereafter referred to as JQVY06) has focused on the relation between the two mechanisms for SSAs in a physical process. As examples, the SIDIS and Drell-Yan processes were considered in a situation where one has two physical scales: the virtuality of the photon, $Q$, and the transverse momentum of the virtual photon $q_{\perp}$ (in Drell-Yan), or of the final hadron $P_{h \perp}$ (in SIDIS). Then, as was argued in JQVY06, in the regime $\Lambda_{\mathrm{QCD}} \ll q_{\perp}$ (or $\left.P_{h \perp}\right) \ll Q$ both the twist-3 and the TMD formalism should apply, and if both are consistent they should describe the same physics and hence become identical. This indeed was verified by performing the following two calculations: first the SSA was computed within the twist-3 formalism, focusing only on the twist-3 quark-quarkgluon correlation function $T_{F}\left(x_{1}, x_{2}\right)$ (the so-called Qiu-Sterman matrix element, with $x_{1}$ and $x_{2}$ the light-cone momentum fractions of the two quarks), and on the contributions from soft-gluon poles (SGPs) and hard poles (HPs) in the corresponding hard-scattering function. Secondly, the Sivers function was calculated to first order in perturbation theory, also in terms of the $T_{F}\left(x_{1}, x_{2}\right)$ matrix element and from the SGP and HP contributions. The result was inserted into TMD factorization formula to obtain the SSA, which led to 
the same answer as that found in the twist-3 approach for $\Lambda_{\mathrm{QCD}} \ll q_{\perp}\left(\right.$ or $\left.P_{h \perp}\right) \ll Q$. In this sense, the two formalisms are unified, providing a unique framework for the SSAs in Drell-Yan and SIDIS.

The twist-3 formalism for SSAs was recently revisited in much detail in Refs. [6, 7] (hereafter referred to as EKT06). Several important issues were clarified and a more complete formula for the SSA in SIDIS was derived. Gauge-invariance and factorization of the twist-3 single-spin-dependent cross section were proved to lowest order. Also the contribution from gluon fragmentation was computed. According to EKT06, there are two types of twist-3 quark-gluon correlation functions in the nucleon that contribute to the single-spin-dependent cross section, $G_{F}\left(x_{1}, x_{2}\right)$ and $\widetilde{G}_{F}\left(x_{1}, x_{2}\right)$. These are defined by

$$
\begin{aligned}
& \int \frac{d \lambda}{2 \pi} \int \frac{d \mu}{2 \pi} e^{i \lambda x_{1}} e^{i \mu\left(x_{2}-x_{1}\right)}\left\langle p S_{\perp}\left|\bar{\psi}_{j}(0) g F^{\alpha \beta}(\mu n) n_{\beta} \psi_{i}(\lambda n)\right| p S_{\perp}\right\rangle \\
& \quad=\frac{M_{N}}{4}(\not p)_{i j} \epsilon^{\alpha p n S_{\perp}} G_{F}\left(x_{1}, x_{2}\right)+i \frac{M_{N}}{4}\left(\gamma_{5} \not p\right)_{i j} S_{\perp}^{\alpha} \widetilde{G}_{F}\left(x_{1}, x_{2}\right)+\cdots
\end{aligned}
$$

where $M_{N}$ is the nucleon mass, $\psi$ is the quark field, $n^{\mu}$ is a light-like vector $\left(n^{2}=0\right)$ with $p \cdot n=1, \epsilon^{\alpha p n S_{\perp}}=\epsilon^{\alpha \lambda \mu \nu} p_{\lambda} n_{\mu} S_{\perp \nu}$ with $\epsilon_{0123}=1$, and the transverse spin vector satisfies $S_{\perp}^{2}=-1, S_{\perp} \cdot p=S_{\perp} \cdot n=0$. We have suppressed the gauge-links that connect the fields along the lightcone and make the matrix element in Eq. (1) gauge-invariant. We note that $G_{F}\left(x_{1}, x_{2}\right)$ is related to $T_{F}\left(x_{1}, x_{2}\right)$ of JQVY06 by $T_{F}\left(x_{1}, x_{2}\right)=-\pi M_{N} G_{F}\left(x_{1}, x_{2}\right)$. Two additional functions could be defined from a matrix element similar to that in Eq. (1) in which, however, the gluon field strength tensor is replaced by a covariant derivative. As was shown in Ref. [6], these functions are not independent but may be expressed in terms of $G_{F}$, $\widetilde{G}_{F}$ and the twist-2 helicity distribution function.

The main features of the twist-3 spin-dependent cross section for SIDIS derived in EKT06 can be summarized as follows. (i) Both $G_{F}\left(x_{1}, x_{2}\right)$ and $\widetilde{G}_{F}\left(x_{1}, x_{2}\right)$ contribute; (ii) the SSA may be generated by three kinds of propagator poles in the hard-scattering function: SGPs, soft-fermion poles (SFPs) and HPs. The SGPs are characterized by $x_{1}=x_{2}$ in the functions $G_{F}$ and $\widetilde{G}_{F}$. For the SFPs, one has $x_{1}=0, x_{2} \neq 0$ (or vice versa), and for the HP contributions the two quark momentum fractions are different and non-vanishing. All three types of poles are present in the contributions associated with $G_{F}$. Because of its antisymmetry property, $\widetilde{G}_{F}\left(x_{1}, x_{2}\right)=-\widetilde{G}_{F}\left(x_{2}, x_{1}\right)$, which follows from parity- and time-reversalinvariance of QCD, $\widetilde{G}_{F}(x, x) \equiv 0$, and hence $\widetilde{G}_{F}$ contributes only through SFPs and HPs.

In the light of the results of EKT06 it is important to extend the studies of JQVY06. 
As we mentioned above, only the "dominant" SGP and HP contributions associated with $G_{F}$ were considered in JQVY06 (for which EKT06 found agreement). The SFPs, the parts involving $\widetilde{G}_{F}$, and the gluon fragmentation contributions were not taken into account in JQVY06. For a complete study of the consistency of the two formalisms for SSAs, it is important to investigate if these additional contributions also organize themselves in such a way that they either become suppressed at $q_{\perp} \ll Q$, and hence negligible from the point of view of TMD factorization, or can be absorbed into the TMD Sivers function. For example, one immediate reason for concern might be the fact that there are two independent twist-3 distribution functions, $G_{F}$ and $\widetilde{G}_{F}$, while only one TMD Sivers function appears in the TMD factorization formalism. In this paper, we will address all these issues.

The rest of the paper is organized as follows. In Sec. II, we will first re-examine the softfermion-pole contributions associated with $G_{F}$ in the twist-3 mechanism for SIDIS. We will show that there are more diagrams than considered in EKT06 that contribute to the SFPs, and we will discuss the effects of these diagrams. We then turn to the contributions associated with $\widetilde{G}_{F}$. After that, we present the single-spin-dependent cross section in the regime $P_{h \perp} \ll Q$, taking into account all $G_{F}$ and $\widetilde{G}_{F}$ contributions in both the quark/antiquark and gluon fragmentation channels. In Sec. III, we will present the "perturbative" one-loop Sivers function in the region $\Lambda_{\mathrm{QCD}} \ll k_{\perp} \ll Q$, including all relevant contributions from $G_{F}$ and $\widetilde{G}_{F}$. Using this Sivers function, the single-spin-dependent cross section in the TMD factorization approach is shown to agree with that in the twist-3 approach. This establishes the true consistency between the TMD and twist-3 formalisms in SIDIS and verifies the original conclusion of [20]. In Sec. IV, we summarize our results.

\section{TWIST-3 CALCULATION}

In EKT06, the SIDIS cross section was calculated for the full kinematic region of transverse momentum, $P_{h \perp} \gg \Lambda_{\mathrm{QCD}}$. The twist-3 single spin-dependent cross section was shown to have the following azimuthal dependence:

$$
d \Delta \sigma \sim \sin \left(\phi_{h}-\phi_{S}\right)\left[\hat{\sigma}_{1}+\hat{\sigma}_{2} \cos \phi_{h}+\hat{\sigma}_{3} \cos 2 \phi_{h}\right]
$$

where $\phi_{h}$ and $\phi_{S}$ are the azimuthal angles of the hadron plane and the transverse spin vector of the nucleon, respectively, measured from the lepton plane in a frame where the 
initial proton and virtual photon are collinear. The leading contribution associated with the Sivers functions gives rise only to the term proportional to $\hat{\sigma}_{1}$ in $(2)$. We also note that the terms associated with $\hat{\sigma}_{2}$ and $\hat{\sigma}_{3}$ in the twist-three cross section are power-suppressed relative to the $\hat{\sigma}_{1}$-term in the small $P_{h \perp} / Q$ region $[6,7]$. This remains true also when the SFP contributions are taken into account. In the following analysis, we will focus only on the leading $\left(\hat{\sigma}_{1}\right)$ contribution with respect to $P_{h \perp} / Q$, which is the relevant contribution for making contact with the TMD formalism.

\section{A. Soft-fermion-pole contributions associated with $G_{F}$}

As was shown in EKT06, both $G_{F}\left(x_{1}, x_{2}\right)$ and $\widetilde{G}_{F}\left(x_{1}, x_{2}\right)$ can give rise to a SSA in SIDIS when combined with the phase from the on-shell part of an internal propagator:

$$
\frac{1}{x_{i} \pm i \varepsilon}=P \frac{1}{x_{i}} \mp i \pi \delta\left(x_{i}\right)
$$

where $x_{i}(i=1$ or 2$)$ is the light-cone momentum fraction carried by one of the quark lines as defined in (1). The pole part of this propagator leads to terms involving $G_{F}(x, 0)$ and $\widetilde{G}_{F}(x, 0)$. The fact that one of the fermion lines carries no longitudinal momentum is the reason why the associated contributions are called soft-fermion-pole (SFP) contributions. In this subsection, we will only discuss the contributions associated with $G_{F}$. The case for $\widetilde{G}_{F}$ will be treated in the next subsection; in any case the discussion is essentially the same for $G_{F}$ and $\widetilde{G}_{F}$. The SFPs appear both in the quark and the gluon fragmentation channels. In the following, we will discuss these separately.

\section{Quark/antiquark fragmentation contributions}

These contributions were calculated from the diagrams in Fig. 6 of EKT06, and the

result for the $G_{F}$ contribution is given in Eq. (84) of their paper [7], and that for the $\widetilde{G}_{F}$ contribution in Eq. (92). Reversing the direction of the fermion lines in these diagrams, one obtains the diagrams for the SFP contributions in the antiquark fragmentation channel, which were also included in the cross section formula of EKT06.

Careful scrutiny of the diagrams leads us to find two other sets of diagrams that also give rise to SFP contributions. One set, for the quark-fragmentation channel, is shown in Fig. 1. 

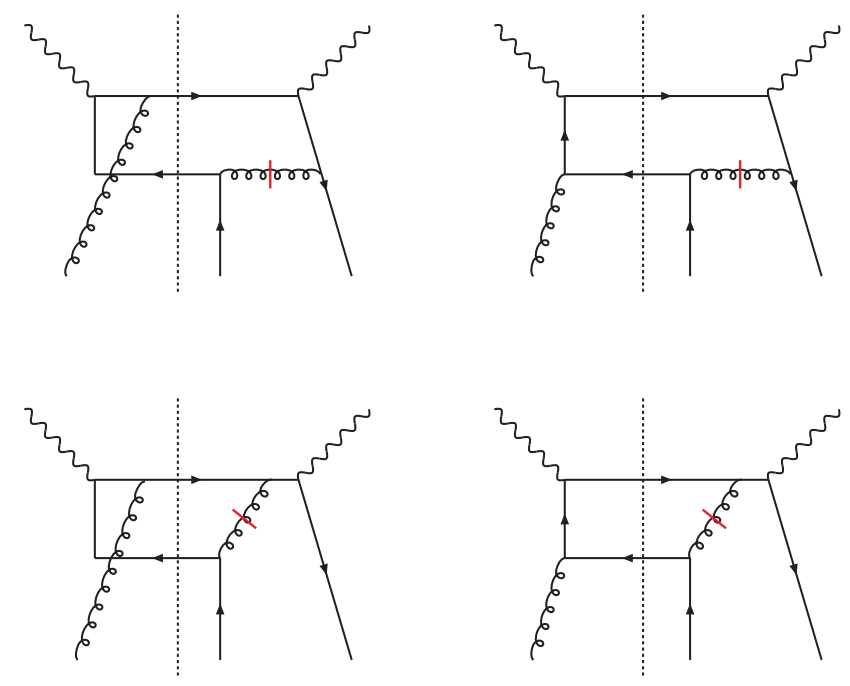

FIG. 1: Additional diagrams contributing to the soft-fermion-pole contributions to the SSA in SIDIS for the quark fragmentation channel. Their contributions exactly cancel those of Fig. 6 of EKT06. Four more diagrams are obtained by reversing the direction of the fermion line and interchanging the momentum labels for the quark lines (see below). For those diagrams, the lower of the two quark lines going through the cut represents the fragmenting quark.

In these diagrams, both the quark- and the antiquark line cross the final-state cut and enter the nucleon matrix element on the right side of the cut. Despite that, the diagrams give an SFP contribution to the cross section with the same matrix elements as those in Fig. 6 of EKT06. They therefore have to be included in the calculation. It is not difficult to find that the contributions by the diagrams in Fig. 1 exactly cancel those from Fig. 6 of EKT06. In order to better understand this cancellation, we redraw in Fig. 2 the upper right diagrams of Fig. 1 and of Fig. 6 of EKT06. The propagator giving the pole is indicated by a short bar in each case. As one can see, these two diagrams are topologically identical, and the only difference between them is the position of the final-state cut and of the SFP. The scattering amplitude for the diagram on the left has the following structure:

$$
\text { Left : } \mathcal{H}\left(x_{1}, x_{2}\right) \frac{1}{\left(k_{2}^{\prime}\right)^{2}+i \epsilon} \delta\left(\left(k_{1}^{\prime}\right)^{2}\right)
$$

where the momenta $k_{1}^{\prime}, k_{2}^{\prime}$ are as labelled in the figure. For the right diagram, one has

$$
\text { Right : } \mathcal{H}\left(x_{1}, x_{2}\right) \frac{1}{\left(k_{1}^{\prime}\right)^{2}-i \epsilon} \delta\left(\left(k_{2}^{\prime}\right)^{2}\right)
$$

When the propagator pole is taken, these two contributions cancel. The other diagrams in 

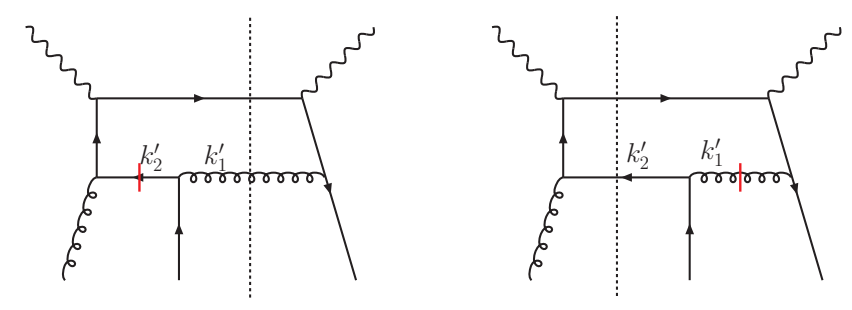

FIG. 2: An example of the cancellation between the diagrams in EKT06 and those in Fig. 1. For the quark fragmentation contribution, the two diagrams will cancel because the soft-fermion attachment is to the "unobserved" final-state particle. The contributions of the diagrams for gluon or antiquark fragmentation do not cancel, however, but are power-suppressed in the limit $P_{h \perp} \ll Q$.

Fig. 6 of EKT06 can be drawn in the same manner, and one can check that they are each cancelled by the corresponding diagram in Fig. 1 .

We note that, in the two diagrams in Fig. 2, the soft-fermion attaches to an "unobserved" final-state parton, that is, the parton that does not fragment into the pion. It is instructive to recall that the diagrams for the SGP contribution also cancel when the soft-gluon-line is attached to an unobserved final parton line [4]. For the SGP this cancellation was clear, because the two cancelling diagrams are just mirror images of each other, with an opposite sign arising from the fact that the soft gluon attaches on different sides of the cut. Here we have found that this cancellation also occurs for the SFP contributions, despite the fact that the two diagrams in Fig. 2 are not mirror images of each other.

A further set of diagrams giving rise to SFP contributions is obtained by reversing the direction of the fermion lines in Fig. 1, along with an interchange of the assignment of the momenta for the external fermion lines, so that now the other final-state line represents the quark that fragments into the final pion. It turns out, however, that this contribution is power-suppressed in the limit $P_{h \perp} \ll Q$.

The discussion for the antiquark-fragmentation channel is completely parallel to that for the quark-fragmentation channel above. As for quark fragmentation there are three types of diagrams, two of which cancel, while the remaining one is power-suppressed for $P_{h \perp} \ll Q$. 


\section{Gluon fragmentation contribution}

For the gluon fragmentation channel, the diagrams in Fig. 6 of EKT06 (with appropriate momentum assignment) are the only ones that give rise to SFP contributions. The corresponding partonic hard cross sections are given in Eq. (89) of [7] for the $G_{F}$ contribution, and in Eq. (94) for the $\widetilde{G}_{F}$ one. As it turns out, in the limit $P_{h \perp} \ll Q$, these contributions are power-suppressed, as inspection of Eqs. (89) and (94) of [7] shows. To obtain this result, one uses the expansion

$$
\begin{aligned}
\delta\left(\frac{\vec{q}_{\perp}^{2}}{Q^{2}}-\right. & \left.\left(1-\frac{1}{\xi}\right)\left(1-\frac{1}{\hat{\xi}}\right)\right) \\
& \rightarrow\left\{\frac{\hat{\xi} \delta(\xi-1)}{(1-\hat{\xi})_{+}}+\frac{\xi \delta(\hat{\xi}-1)}{(1-\xi)_{+}}+\delta(\xi-1) \delta(\hat{\xi}-1) \ln \frac{Q^{2}}{\vec{q}_{\perp}^{2}}\right\},
\end{aligned}
$$

for the phase-space delta function relevant for SIDIS, which is valid for $\vec{q}_{\perp}^{2} \ll Q^{2}$. Here $\vec{q}_{\perp}=-\vec{P}_{h \perp} / z_{h}, \xi=\hat{x}=x_{B} / x$ and $\hat{\xi}=\hat{z}=z_{h} / z$, as in EKT06. We note that the first term in this expansion corresponds to contributions that can be regarded as part of the TMD fragmentation function, while the second term leads to terms to be absorbed into the quark Sivers function. These observations will be useful later when making contact with the TMD formalism. Substituting the above expansion into the cross section formulas given in EKT06, we straightforwardly find that the gluon fragmentation terms from SFPs vanish in the limit $P_{h \perp} \ll Q$.

On the other hand, for gluon fragmentation there are SGP and HP contributions associated with $G_{F}$ that survive in the limit $P_{h \perp} \ll Q$. Their contributions can be derived from the results in Eqs. (87),(88) of [7] by applying the above expansion for the delta function. We will include these terms in our final results to be presented later.

\section{B. Contributions associated with $\widetilde{G}_{F}$}

A possibly important contribution not considered in [18-20] comes from the other twist-3 distribution $\widetilde{G}_{F}\left(x_{1}, x_{2}\right)$ defined in (1). $\widetilde{G}_{F}$ is antisymmetric in its arguments [4, 7], and hence $\widetilde{G}_{F}(x, x) \equiv 0$. Therefore, there are no SGP contributions to the single-spin-dependent

cross section associated with $\widetilde{G}_{F}$. However, as was shown in EKT06, $\widetilde{G}_{F}$ does give rise to SFP and HP contributions. Regarding the former, the situation in the quark/antiquark fragmentation channel is the same as for $G_{F}$. After inclusion of the contributions from 
the additional diagrams missing in EKT06, there is no surviving SFP contribution in the $P_{h \perp} \ll Q$ limit.

The HP contributions for $\widetilde{G}_{F}$ come from the same diagrams as for $G_{F}$; see [20] and Fig. 2 of [7]. The results can be found in Eq. (91) of [7] for the quark-fragmentation channel and in Eq. (93) for the gluon fragmentation channel. As for the $G_{F}$ case, the contributions involve $\widetilde{G}_{F}\left(x_{B}, x\right)$, with one variable equal to Bjorken- $x_{B}$. In the limit $P_{h \perp} \ll Q$ we use again the expansion in (6) and only consider the leading-power contribution. The first and the third terms of the expansion do not contribute, because $\delta(\xi-1)$ means that $x=x_{B}$, which leads to $\widetilde{G}_{F}\left(x_{B}, x_{B}\right) \equiv 0$. Thus, we will only have contributions from the $\delta(\hat{\xi}-1)$ term in $(6)$. These can be factorized into the quark Sivers function.

\section{Twist-3 cross section in the $P_{h \perp} \ll Q$ limit}

We are now in the position to present the twist-3 single-spin-dependent cross section in the regime $\Lambda_{\mathrm{QCD}} \ll P_{h \perp} \ll Q$, including all the surviving contributions by $G_{F}$ and $\widetilde{G}_{F}$. It reads

$$
\begin{aligned}
\frac{d \Delta \sigma\left(S_{\perp}\right)}{d x_{B} d y d z_{h} d^{2} \vec{P}_{h \perp}}= & -\frac{4 \pi \alpha_{\mathrm{em}}^{2} S_{e p}}{Q^{4}} \epsilon_{\alpha \beta} S_{\perp}^{\alpha} \frac{z_{h} P_{h \perp}^{\beta}}{\left(\vec{P}_{h \perp}^{2}\right)^{2}} \frac{\alpha_{s}}{2 \pi^{2}} x_{B}\left(1-y+\frac{y^{2}}{2}\right) \int \frac{d x d z}{x z}\left\{D_{q}(z)\right. \\
& \left.\times[\delta(\hat{\xi}-1) A+\delta(\xi-1) B]+C_{F} \delta(\xi-1) T_{F}(x, x) D_{g}(z) \frac{1+(1-\hat{\xi})^{2}}{\hat{\xi}}\right\},
\end{aligned}
$$

where

$$
\begin{aligned}
A= & \frac{1}{2 N_{C}}\left\{\left[x \frac{\partial}{\partial x} T_{F}(x, x)\right]\left(1+\xi^{2}\right)+T_{F}\left(x, x-\widehat{x}_{g}\right) \frac{1+\xi}{(1-\xi)_{+}}\right. \\
& \left.+T_{F}(x, x) \frac{(1-\xi)^{2}(2 \xi+1)-2}{(1-\xi)_{+}}\right\}+C_{F} T_{F}\left(x, x-\widehat{x}_{g}\right) \frac{1+\xi}{(1-\xi)_{+}} \\
& +\left(C_{F}+\frac{1}{2 N_{c}}\right) \widetilde{T}_{F}\left(x-\widehat{x}_{g}, x\right), \\
B= & C_{F} T_{F}(x, x)\left[\frac{1+\hat{\xi}^{2}}{(1-\hat{\xi})_{+}}+2 \delta(\hat{\xi}-1) \ln \frac{z_{h}^{2} Q^{2}}{\vec{P}_{h \perp}^{2}}\right],
\end{aligned}
$$

where $\widehat{x}_{g} \equiv(1-\xi) x=x-x_{B}$, and we have used the notation $\widetilde{T}_{F}\left(x_{1}, x_{2}\right) \equiv-\pi M_{N} \widetilde{G}_{F}\left(x_{1}, x_{2}\right)$ as well as $T_{F}\left(x_{1}, x_{2}\right)=-\pi M_{N} G_{F}\left(x_{1}, x_{2}\right)$ to make the comparison with Ref. [20] (Eq. (18)) 
manifest. $D_{q}$ and $D_{g}$ are the usual transverse-momentum integrated quark and gluon fragmentation functions, respectively. Eq. (7) extends the results in Eq. (18) of [20] through the inclusion of the $\widetilde{G}_{F}$-term in (8) and the term involving $D_{g}$. The $B$ term in Eq. (9) remains the same as that in [20]. This is crucial because this term corresponds to a contribution by near-collinear gluon emission from the unpolarized outgoing quark, which is completely determined [12]. All other contributions in EKT06 are power-suppressed in the limit $P_{h \perp} \ll Q$.

To fully establish the consistency between the twist-3 and the TMD factorization approaches in the intermediate region of the transverse momentum, we now need to show that the result in Eq. (7) is reproduced in the TMD formalism. In particular, one needs to address

the issue that there are two independent twist-3 distribution functions, $G_{F}$ and $\widetilde{G}_{F}$, in the transversely polarized nucleon, while only one TMD Sivers function appears in the TMD factorization. We will see in the next section that there are also additional contributions to the Sivers function that restore the equivalence of the two formalisms for $\Lambda_{\mathrm{QCD}} \ll P_{h \perp} \ll Q$.

\section{CONSISTENCY WITH THE TMD APPROACH}

As was shown in $[19,20]$, in the regime $\Lambda_{\mathrm{QCD}} \ll k_{\perp}, p_{\perp} \ll Q$ the TMD quark Sivers function $q_{T}\left(x, k_{\perp}\right)$ and the TMD quark fragmentation function $\hat{q}\left(z, p_{\perp}\right)$ can be calculated perturbatively. At the first order, one finds for the TMD quark fragmentation function [20]:

$$
\begin{aligned}
\hat{q}\left(z_{h}, p_{\perp}\right)= & \frac{\alpha_{s}}{2 \pi^{2}} \frac{1}{\vec{p}_{\perp}^{2}} C_{F} \int \frac{d z}{z}\left\{D_{q}(z)\left[\frac{1+\hat{\xi}^{2}}{(1-\hat{\xi})_{+}}+\delta(\hat{\xi}-1)\left(\ln \frac{\hat{\zeta}^{2}}{\vec{p}_{\perp}^{2}}-1\right)\right]\right. \\
& \left.+D_{g}(z) \frac{1+(1-\hat{\xi})^{2}}{\hat{\xi}}\right\},
\end{aligned}
$$

where $D_{q}(z)$ and $D_{g}(z)$ are again the integrated quark and gluon fragmentation functions. The $D_{g}$-term in this equation is new compared to [20]; it comes from the radiation of a gluon which then fragments.

Similarly, the TMD quark Sivers function $q_{T}\left(x, k_{\perp}\right)$ can be calculated [19]. The crucial point for our present discussion is that it receives contributions not only from $G_{F}$ (which were calculated in [19]), but also by $\widetilde{G}_{F}$. These can be calculated following the techniques given in [19]. The relevant diagrams are the same as those in Fig. 10 of [19], which were given there for the HP contributions for $G_{F}$. One only needs to insert the appropriate projection 
matrix for $\widetilde{G}_{F}$ as seen in Eq. (1). Combining all contributions, one finds:

$$
q_{T}\left(x_{B}, k_{\perp}\right)=-\frac{\alpha_{s}}{4 \pi^{2}} \frac{2 M_{N}}{\left(\vec{k}_{\perp}^{2}\right)^{2}} \int \frac{d x}{x}\left\{A+C_{F} T_{F}(x, x) \delta(\xi-1)\left(\ln \frac{x_{B}^{2} \zeta^{2}}{\vec{k}_{\perp}^{2}}-1\right)\right\},
$$

where $A$ has been defined in Eq. (8) and contains the function $\widetilde{G}_{F}$.

Substituting the above expressions for the TMD quark Sivers function and the TMD quark fragmentation function, along with the soft factor calculated in [18-20], into the TMD factorization formula [12], one reproduces the differential cross section in Eq. (7) obtained from the twist-3 quark-gluon correlation approach in the intermediate-transverse-momentum region $\Lambda_{\mathrm{QCD}} \ll P_{h \perp} \ll Q$. This demonstrates the full consistency of these two approaches in SIDIS.

We close this section with two further observations. First, we emphasize that the new contribution to the quark Sivers function associated with $\widetilde{G}_{F}$ still obeys the well-known non-universality property of the quark Sivers function. In particular, it will change sign for the Drell-Yan process [18-20].

Secondly, the above result shows that the perturbative calculation of $G_{F}$ receives contributions both from $G_{F}$ and $\widetilde{G}_{F}$. This is a consequence of the fact that $G_{F}$ and $\widetilde{G}_{F} \operatorname{mix}$ under renormalization, as is well known in the context of the renormalization of the twist-3 part of the $g_{2}$ structure function which can be represented by the same two functions [21].

\section{SUMMARY}

We have revisited the relation uncovered in $[18,19]$ between the twist-3 and the TMD formalisms for the single-transverse-spin asymmetry in semi-inclusive lepton scattering. We have considered additional contributions in the twist-3 formalism, which were recently derived in [7]. These contributions are associated with soft-fermion poles, with another twist-3 correlation function, termed $\widetilde{G}_{F}$ in [7], and with gluon fragmentation. We have investigated the behavior of these contributions in the limit $P_{h \perp} \ll Q$ and verified that the new contributions organize themselves in a way consistent with the transverse momentum dependent factorization formalism. An important ingredient to this is that the quark Sivers function itself receives contributions from the twist-3 quark gluon correlation function $\widetilde{G}_{F}$, in addition to that from the previously considered Qiu-Sterman matrix element $G_{F}$. This also indicates that $G_{F}$ and $\widetilde{G}_{F}$ will mix under renormalization. 
Thus, there is full equivalence of the two formalisms in the intermediate-transversemomentum regime. All our calculations can be straightforwardly extended to the SSA in the Drell-Yan process $[18,19]$, and we find that the consistency between the two approaches remains intact also here.

We thank Alessandro Bacchetta and Markus Diehl for bringing to our attention the issue of consistency of the two formalisms in the light of the soft-fermion-pole and $\widetilde{G}_{F}$ contributions derived in EKT06. Y.K. thanks K. Tanaka for a useful discussion, and F.Y. thanks X.N. Wang for a useful conversation. This work was supported in part by the U.S. Department of Energy under grant contract DE-AC02-05CH11231. F.Y. and W.V. thank RIKEN, Brookhaven National Laboratory and the U.S. Department of Energy (contract number DE-AC02-98CH10886) for providing the facilities essential for the completion of their work.

[1] D. W. Sivers, Phys. Rev. D 41, 83 (1990); Phys. Rev. D 43, 261 (1991).

[2] J. C. Collins, Nucl. Phys. B 396, 161 (1993).

[3] A. V. Efremov and O. V. Teryaev, Sov. J. Nucl. Phys. 36, 140 (1982) [Yad. Fiz. 36, 242 (1982)]; A. V. Efremov and O. V. Teryaev, Phys. Lett. B 150, 383 (1985).

[4] J.W. Qiu and G. Sterman, Phys. Rev. Lett. 67, 2264 (1991); Nucl. Phys. B 378, 52 (1992); Phys. Rev. D 59, 014004 (1998).

[5] Y. Kanazawa and Y. Koike, Phys. Lett. B 478, 121 (2000); Phys. Rev. D 64, 034019 (2001).

[6] H. Eguchi, Y. Koike and K. Tanaka, Nucl. Phys. B 752, 1 (2006).

[7] H. Eguchi, Y. Koike and K. Tanaka, Nucl. Phys. B 763, 198 (2007).

[8] Y. Koike, AIP Conf. Proc. 675, 449 (2003).

[9] S. J. Brodsky, D. S. Hwang and I. Schmidt, Phys. Lett. B 530, 99 (2002); Nucl. Phys. B 642, 344 (2002).

[10] J. C. Collins, Phys. Lett. B 536, 43 (2002).

[11] X. Ji and F. Yuan, Phys. Lett. B 543, 66 (2002); A. V. Belitsky, X. Ji and F. Yuan, Nucl. Phys. B 656, 165 (2003).

[12] X. Ji, J. P. Ma and F. Yuan, Phys. Rev. D 71, 034005 (2005); Phys. Lett. B 597, 299 (2004). 
[13] J. C. Collins and A. Metz, Phys. Rev. Lett. 93, 252001 (2004).

[14] D. Boer, P. J. Mulders and F. Pijlman, Nucl. Phys. B 667, 201 (2003).

[15] C. J. Bomhof, P. J. Mulders and F. Pijlman, Phys. Lett. B 596, 277 (2004); Eur. Phys. J. C 47, 147 (2006); JHEP 0702, 029 (2007); A. Bacchetta, C. J. Bomhof, P. J. Mulders and F. Pijlman, Phys. Rev. D 72, 034030 (2005); C. J. Bomhof and P. J. Mulders, arXiv:0709.1390 [hep-ph].

[16] J. W. Qiu, W. Vogelsang and F. Yuan, Phys. Lett. B 650, 373 (2007); Phys. Rev. D 76, 074029 (2007); W. Vogelsang and F. Yuan, arXiv:0708.4398 [hep-ph].

[17] J. Collins and J. W. Qiu, Phys. Rev. D 75, 114014 (2007); J. Collins, arXiv:0708.4410 [hep-ph].

[18] X. Ji, J. W. Qiu, W. Vogelsang and F. Yuan, Phys. Rev. Lett. 97, 082002 (2006).

[19] X. Ji, J. W. Qiu, W. Vogelsang and F. Yuan, Phys. Rev. D 73, 094017 (2006).

[20] X. Ji, J. W. Qiu, W. Vogelsang and F. Yuan, Phys. Lett. B 638, 178 (2006).

[21] See, for example: I. I. Balitsky and V. M. Braun, Nucl. Phys. B 311, 541 (1989); P. G. Ratcliffe, Nucl. Phys. B 264, 493 (1986); X. D. Ji and C. h. Chou, Phys. Rev. D 42, 3637 (1990); J. Kodaira, Y. Yasui, K. Tanaka and T. Uematsu, Phys. Lett. B 387, 855 (1996); A. V. Belitsky, X. D. Ji, W. Lu and J. Osborne, Phys. Rev. D 63, 094012 (2001). 